УДК 676.054.7

\title{
ОБ ЭФФЕКТИВНОСТИ ПРИМЕНЕНИЯ АППАРАТА ПРОФИЛИРОВАННОЙ ФОРМЫ С РОТОРОМ ГЕЛИКОИДАЛЬНОГО
} ТИПА В ЦБП

\author{
() Д.А. Иванов", К.А. Иванов, А.П. Руденко \\ Сибирский государственный технологический университет, пр. Мира, 82, \\ Красноярск, 660049 (Россия), e-mail: ivanov.sibstu@yandex.ru
}

Представлены экспериментальные исследования гидродинамической картины движения потоков жидкости в перемешивающих аппаратах цилиндрической и профилированной формы. Показана эффективность применения аппарата профилированной формы с ротором геликоидального типа при перемешивании волокнистых суспензий различной концентрации.

Ключевые слова: гидродинамика, гомогенность, корпус, направляющий аппарат, полость, профилирование, ротор, суспензия, циркуляция.

Развитие ЦБП и смежных отраслей, где используются процессы перемешивания, во многом определяется совершенствованием перемешивающего оборудования, например таких, как мешальные (машинные) бассейны, которые предназначены не только для создания запаса массы, но и для выравнивания композиции и концентрации массы при помощи встроенных в них перемешивающих устройств $[1,2]$.

В настоящий момент применяемые перемешивающие аппараты характеризуются относительно невысокой удельной производительностью процессов перемешивания по целевому продукту в силу проявления не идеальных по объему аппарата условий перемешивания среды, различием массообменных характеристик по зонам аппарата, тепловой и диффузионной неравномерностью. Несоответствие конструктивного исполнения аппарата и выполняемого процесса перемешивания является одной из причин низкой энергетической эффективности такого оборудования, наличия слабо перемешиваемых застойных зон в проточной полости аппарата и неустойчивости протекания самого процесса. По этой причине в таких аппаратах не удается достичь равномерного распределения подводимой энергии, что приводит к повышенным энергозатратам в процессе получения целевого продукта, а следовательно, и к повышению его себестоимости.

Как известно, рыночная экономика требует повышения качества, расширения ассортимента выпускаемых продуктов, что вызывает необходимость модернизации старых и внедрения новых, прогрессивных технологий и более совершенного оборудования. Поэтому важным направлением научно-технических исследований является разработка принципиально новых конструкций машин и методов их инженерного расчета, позволяющих существенно снизить энергозатраты, в первую очередь на перемешивание - опера-

Иванов Дмитрий Александрович - аспирант, e-mail: ivanov.sibstu@yandex.ru

Иванов Кирилл Александрович - аспирант, e-mail: www.kir_ill@list.ru

Руденко Анатолий Павлович - заведующий кафедрой технологии конструкционных материалов

и машиностроения, доктор технических наук, профессор, тел.: (3912) 12-23-36,

e-mail: tolyrudenko@yandex.ru цию, которая присутствует в преобладающем большинстве технологических линий по получению готового продукта.

Наличие данного обстоятельства порождает необходимость поиска новых, прогрессивных конструктивных решений относительно корпусного выполнения перемешивающих устройств с новым типом перемешивающих органов в целлюлозно-

\footnotetext{
* Автор, с которым следует вести переписку.
} 


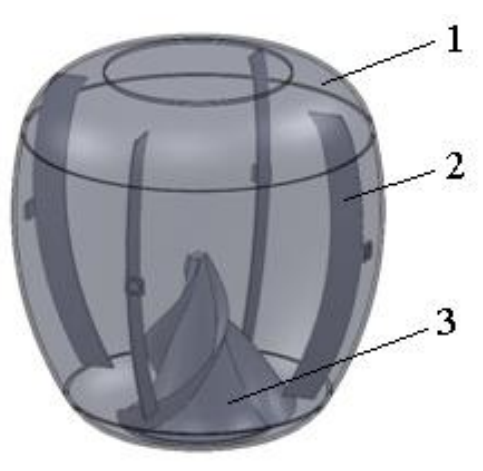

Рис. 1. Аппарат

профилированного корпуса с ротором геликоидного типа:

1 - профилированный корпус;

2 - отражательная планка;

3 - ротор геликоидального типа

бумажной промышленности для конструктивного исполнения перемешивающих аппаратов и емкостей различного функционального назначения (мешальные бассейны, смесители, хлораторы и т.п.).

Для осуществления этих целей в Сибирском государственном технологическом университете продолжаются научноисследовательские работы в данной области. На основе теоретических исследований с применением программы имитационного моделирования Solid Works [3] был разработан аппарат с профилированными элементами корпуса с ротором геликоидального типа (рис. 1).

С целью определения интенсивности проведения процессов перемешивания в предлагаемом аппарате по сравнению с перемешивающими аппаратами с цилиндрическим корпусом были проведены экспериментальные исследования гидродинамики, основанные на зондировании проточной полости перемешивающих аппаратов посредством измерительно-вычислительного комплекса ИВК [4]. При этом особое внимание уделялось соотношению окружных и осевых скоростей потока жидкости, существенным образом влияющих на качество перемешивания. Так, при преобладании осевой скорости потока над окружной скоростью степень циркуляции жидкости возрастает. С увеличением степени циркуляции возрастает интенсивность перемешивания, а значит, повышается качество целевого продукта [5].

Экспериментальные исследования гидродинамики в проточной полости перемешивающих аппаратов посредством ИВК показали, что применение перемешивающего аппарата профилированного корпуса привело к снижению роста окружной скорости по высоте аппарата снизу вверх от 2 до 4,8 раза по сравнению с аппаратом цилиндрической формы в зависимости от частоты вращения ротора. Осевые скорости, наоборот, возросли с увеличением высоты аппарата в среднем до 2,3 раза и имеют максимальные значения у стенки корпуса, где окружные скорости минимальны. Это свидетельствует об увеличении степени циркуляции жидкости, а значит, и о минимизации возникновения застойных зон, в том числе центральной застойной зоны, по сравнению с аппаратом цилиндрической формы.

В целом абсолютная скорость в профилированном корпусе по сравнению с цилиндрическим корпусом выросла. При этом наиболее высокий прирост скорости наблюдается при частоте вращения $\mathrm{n}=500$ об/мин и составляет около $36 \%$.

Полученные результаты также показали, что в верхней части проточной полости аппарата с увеличением частоты вращения перемешивающего органа наблюдается смещение траектории движения от оси ротора к периферии при течении потока жидкости сверху вниз. Такое смещение приводит к увеличению пути движения потока и снижению степени циркуляции, что, в свою очередь, отрицательно сказывается на интенсификации самого процесса перемешивании и на качестве получаемого продукта.

Для улучшения гидродинамической картины движения потоков жидкости в верхней части аппарата был реализован и установлен направляющий аппарат [6] (рис. 2). Конструкция направляющего аппарата спроектирована таким образом, чтобы поток жидкости входил в

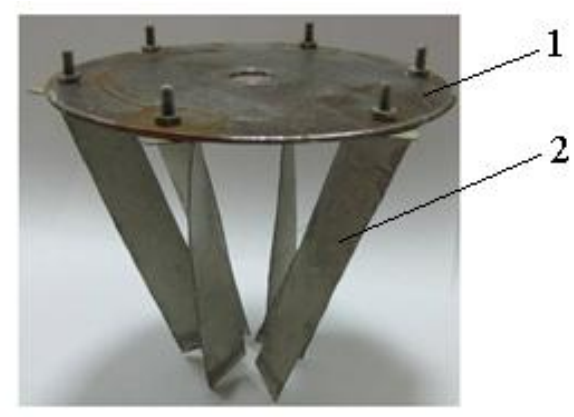

Рис. 2. Общий вид направляющего аппарата: 1 - основание;

2 - направляющая планка него при углах атаки, не превышающих $20^{\circ}$. При таких углах атаки поток обладает безотрывным движением, что позволяет снизить появление вихреобразования в верхней проточной полости аппарата и направить поток сразу к перемешивающему органу. Это, в свою очередь, повышает степень циркуляции и интенсифицирует процесс приготовления гомогенной массы.

Исследования гидродинамики проводились при разных углах установки лопаток направляющего аппарата в горизонтальной плоскости (от 0 до 30 градусов).

По результатам эксперимента были получены аналитические зависимости (1-4) абсолютной скорости движения потоков жидкости и ее составляющих и построены эпюры скоростей (рис. 3-5). 


$$
\begin{gathered}
\vartheta_{\text {абс }}=0,622+0,0014 n+5,095 R+2,459 H+1,088 \operatorname{tg} \alpha+0,0000018 n^{2}- \\
-0,0025 n t g \alpha-43,057 R H-9,566 H \operatorname{tg} \alpha \\
\vartheta_{\text {окр }}=0,3955+0,0052 n-0,0000044 n^{2}-0,016 n H+38,638 R H- \\
-10,152 R \operatorname{tg} \alpha-3,915 \operatorname{tg} \alpha \\
\vartheta_{\text {рад }}=-0,172-2,191 H-4,295 \cdot 10^{-7} n^{2}-0,0068 n R+0,041 n H+ \\
+0,00033 n t g \alpha+24,467 R H+1,113 H \operatorname{tg} \alpha \\
\vartheta_{\text {осев }}=-0,264+13,616 R+4,531 H+1,86 t g \alpha+0,0000017 n^{2}+0,013 n H- \\
-0,0036 n t g \alpha-117,307 R H-8,791 \operatorname{tg} \alpha ;
\end{gathered}
$$

где $n$ - частота вращения ротора, об/мин; $R$ - радиус рассматриваемого сечения в аппарате, м; $H$ - высота рассматриваемого сечения в аппарате, м; $\operatorname{tg} \alpha$ - угол поворота планки в направляющем аппарате.

Анализ полученных результатов показал, что при наличии направляющего аппарата в верхней части аппарата происходит значительное снижение окружной составляющей абсолютной скорости (тах до $25 \%$ при $\alpha=30^{\circ}$ ) и рост осевой составляющей ( $\max$ до $34 \%$ при $\alpha=0^{\circ}$ ). При этом распределение осевых скоростей в верхних рассматриваемых сечениях происходит равномерно (сечения 3-4 рис. 3-5). Также определено, что с увеличением угла установки лопаток в направляющем аппарате вертикальная составляющая абсолютной скорости снижается (до 30\%), что свидетельствует о возникновении эффекта отрывности потока жидкости.

a)

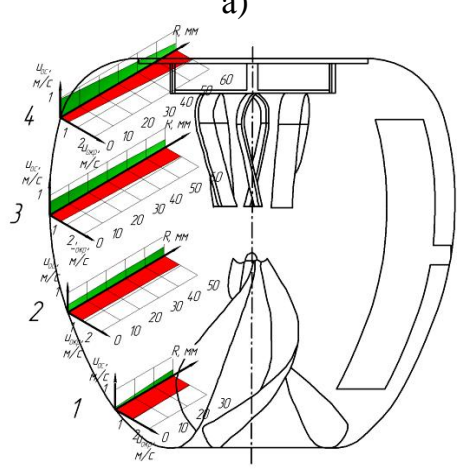

б)

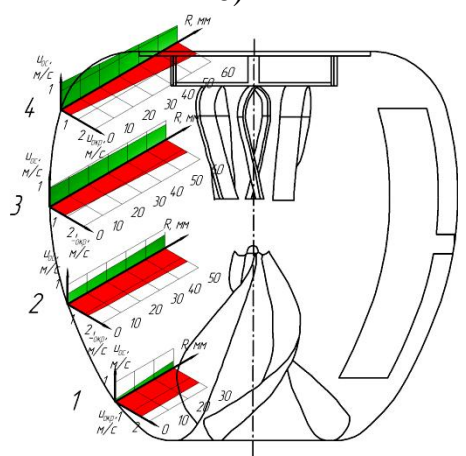

B)

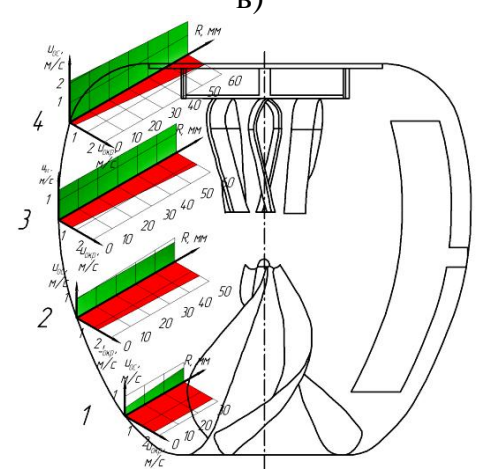

Рис. 3. Схема эпюр скоростей в аппарате профилированного сечения с направляющим аппаратом. Угол установки лопаток в направляющем аппарате $\alpha=0^{\circ}$; a) - n=100 об/мин; б) - 300 об/мин; в) - 500 об/мин. 1, 2, 3, 4 - рассматриваемые сечения в аппарате. Зеленое (светлое) поле - осевые скорости; красное (темное) поле - окружные скорости

a)

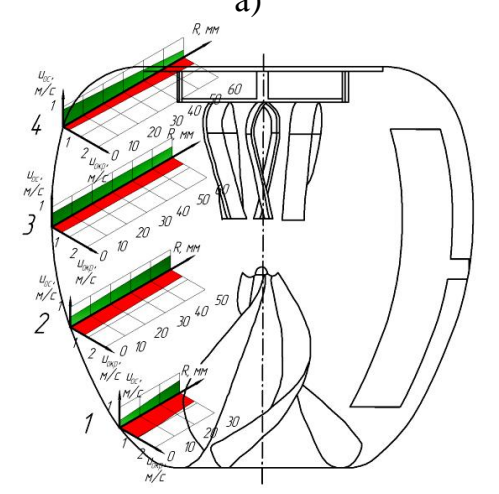

б)

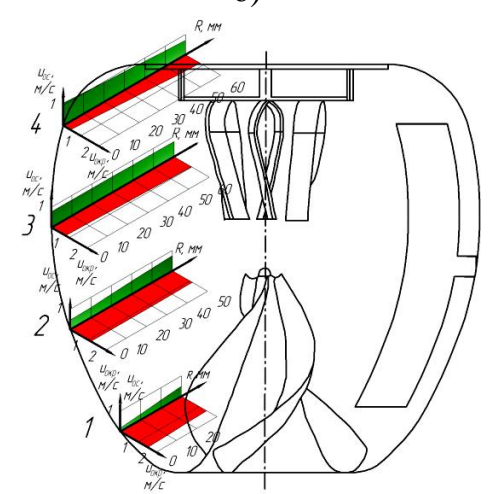

B)

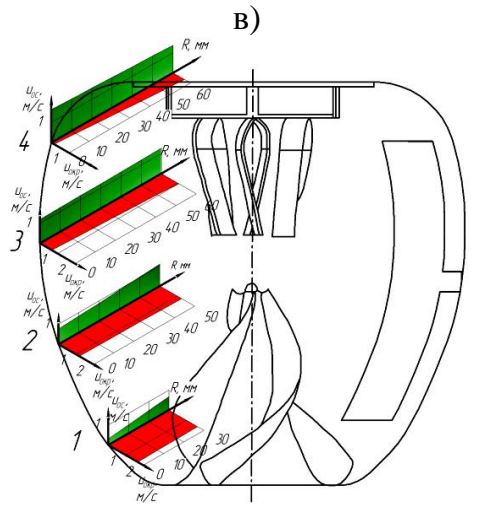

Рис. 4. Схема эпюр скоростей в аппарате профилированного сечения с направляющим аппаратам. Угол установки лопаток в направляющем аппарате $\alpha=15^{\circ}$; a) - n=100 об/мин; б) - 300 об/мин; в) - 500 об/мин. 1, 2, 3, 4 - рассматриваемые сечения в аппарате. Зеленое (светлое) поле - осевые скорости; красное (темное) поле - окружные скорости 
a)

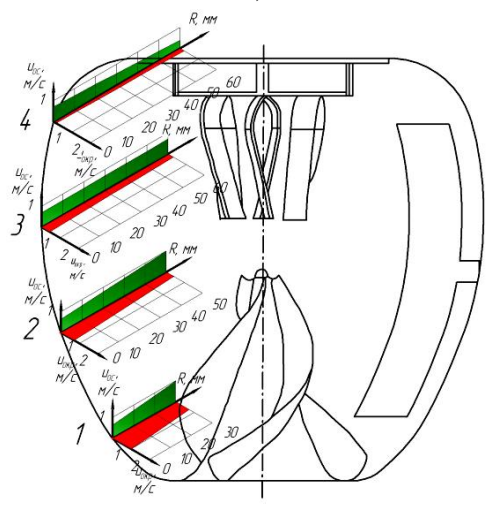

б)

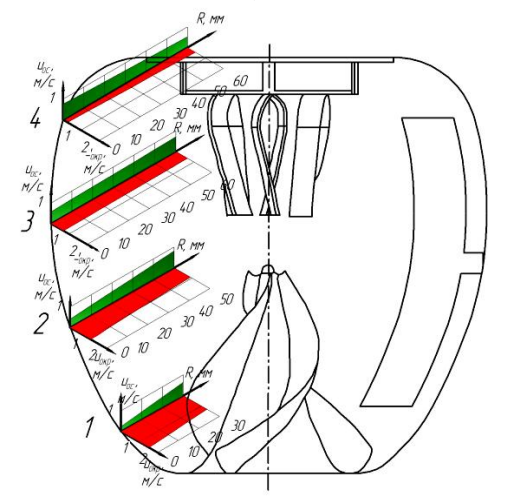

B)

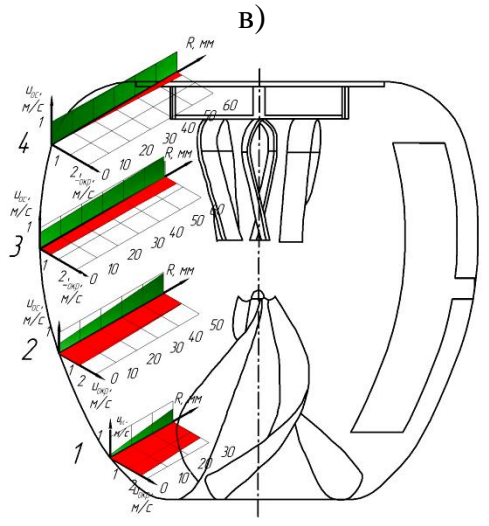

Рис. 5. Схема эпюр скоростей в аппарате профилированного сечения с направляющим аппаратом. Угол установки лопаток в направляющем аппарате $\alpha=30^{\circ}$; a) - n= 100 об/мин; б) - 300 об/мин; в) - 500 об/мин. 1, 2, 3, 4- рассматриваемые сечения в аппарате. Зеленое (светлое) поле - осевые скорости; красное (темное) поле - окружные скорости

Положительный эффект использования направляющего аппарата наблюдается при $\mathrm{n} \geq 300$ об/мин, так как при средних и больших значениях частоты вращения ротора влияние окружной составляющей абсолютной скорости на циркуляцию потока становится значимой. В этом случае имеет место достаточно эффективная трансформация окружной составляющей в осевую скорость с использованием направляющего аппарата.

В целом применение аппарата профилированной формы без направляющего аппарата приводит к росту осевой скорости потока до $25 \%$, а с направляющим аппаратом - до $35 \%$ по сравнению с аппаратом цилиндрической конструкции.

На рисунке 6 представлена зависимость абсолютной скорости движения потока рабочей жидкости от частоты вращения ротора для различных конструкций исследуемых перемешивающих аппаратов. Как следует из графика, наибольшее значение абсолютной скорости имеет место в аппарате профилированной формы с направляющим аппаратом с углом установки лопаток $\alpha=0^{\circ}$, наименьшее - в аппарате с цилиндрическим корпусом.

На основе полученных результатов исследования была выполнена оптимизация технологических параметров, обеспечивающих оптимальные условия проведения процесса перемешивания в аппарате профилированной формы с направляющим аппаратом с углом установки лопаток $\alpha=0^{\circ}$. В результате оптимизации по осевой скорости $\mathrm{u}_{\mathrm{oc}}$ были получены следующие значения оптимизируемых параметров: $\mathrm{n}=471$ об/мин, $\alpha=20^{\circ}$.

В условиях оптимальной работы перемешивающего аппарата с профилированным корпусом и направляющим аппаратом было исследовано влияние концентрации волокнистой суспензии на эффективность работы, причем значения концентрации изменялись в пределах 0,5, 0,8, 1 и 1,5\%.

В результате обработки данных получены аналитические выражения абсолютной скорости движения волокнистой суспензии и ее составляющих, а также построены эпюры скоростей для суспензий указанных выше концентраций.

При анализе эпюр скоростей установлено, что с увеличением концентрации суспензии осевая скорость потока уменьшается по высоте аппарата под действием сил тяжести волокон, но наряду с этим наблюдается ее рост в области, непосредственно примыкающей к ротору.

Зависимость средней осевой составляющей скорости от концентрации волокнистой суспензии представлена на графике (рис. 7).

Как видно из графика, наиболее предпочтительной концентрацией волокнистой суспензии для эффективной работы аппарата профилированной формы на оптимальном режиме является концентрация $0,8-0,9 \%$, при этом осевая скорость принимает значение равное около $1 \mathrm{~m} / \mathrm{c}$. В этом случае поток рабочей жидкости становится диспергированным (происходит полное разрушение структуры потока под действием критического градиента сдвига (турбулентных вихрей)) [7] и отлично перемешивается. При дальнейшем увеличении концентрации волокнистой суспензии поток снова становится структурированным, в результате чего его скорость и эффективность перемешивания падает. 


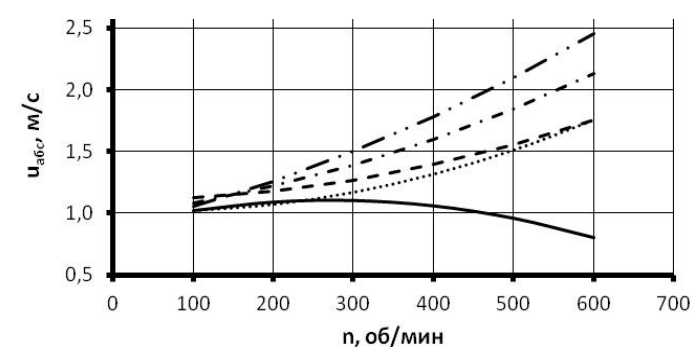

Рис. 6. Зависимость абсолютной скорости от частоты вращения ротора геликоидального типа для различных конструкций перемешивающих аппаратов: 1 - аппарат с цилиндрическим корпусом; 2 - профилированный корпус без направляющего аппарата; 3, 4, 5 - с установкой планок в направляющем аппарате под следующими углами: $3-\alpha=0^{\circ} ; 4-\alpha=15^{\circ} ; 5-\alpha=30^{\circ}$

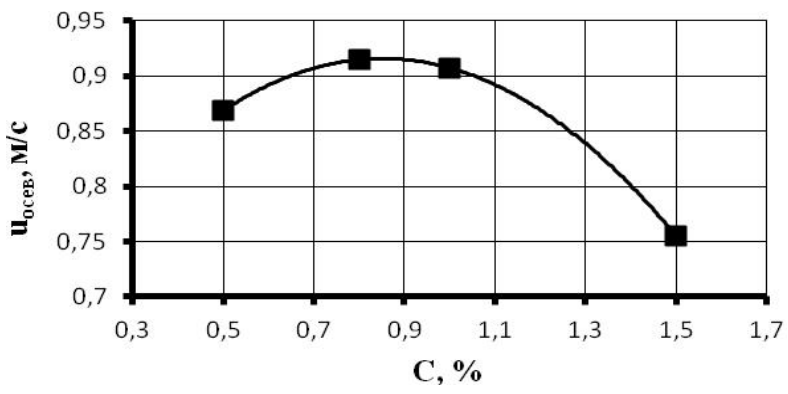

Рис. 7. Зависимость средней осевой составляющей скорости потока от концентрации волокнистой суспензии

При перемешивании волокнистых суспензий с другими концентрациями необходимо произвести корректировку технологических режимов работы аппарата до достижения требуемого эффекта.

\section{Выводы}

1. На основании проведенных исследований и полученных результатов следует отметить, что разработанный аппарат профилированной формы обладает потенциальной возможностью интенсивного перемешивания рабочей жидкости за счет наличия большого диапазона настройки аппарата на требуемый технологический режим.

2. Повышение интенсивности перемешивания позволяет сократить время приготовления целевого продукта, тем самым уменьшая потребляемую энергию. Это, в свою очередь, способствует снижению себестоимости производимого продукта.

\section{Список литературы}

1. Чичаев В.А., Глезин М.Л., Екимова В.А. Оборудование целлюлозно-бумажного производства. В 2 т. Т. 2. Бумагоделательные машины. М., 1981. 264 с.

2. Фляте Д.М. Технология бумаги. Учебник для вузов. М., 1988. 440 с.

3. Иванов Д.А., Иванов К.А., Руденко А.П. Создание многофункциональных емкостных аппаратов с использованием имитационного моделирования // Химия растительного сырья. 2011. №4. С. 319-324.

4. Иванов Д.А., Иванов К.А., Руденко А.П. Диагностика эффективности работы перемешивающих аппаратов с помощью измерительно-вычислительного комплекса // Лесной и химический комплексы - проблемы и решения: сб. статей всерос. науч.-практич. конф. Красноярск, 2012. Т. II. С. 46-50.

5. Стренк Ф. Перемешивание и аппараты с мешалками. Л., 1975. 384 с.

6. Патент 2453589. (РФ). Аппарат для выращивания мицелиальных форм микроорганизмов / А.П. Руденко, Д.А. Иванов, К.А. Иванов. БИ. №17. 20.06.2012.

7. Терентьев О.А. Гидродинамика волокнистых суспензий в целлюлозно-бумажном производстве. М., 1980. 248 с. 
Ivanov K.A., Ivanov D.A., Rudenko A.P. ABOUT EFFICIENCY OF USE OF THE APPARATUS OF PROFILED FORM WITH THE HELICOID TYPE ROTOR IN PPI

Siberian State Technological University, pr. Mira, 82, Krasnoyarsk, 660049 (Russia), e-mail: ivanov.sibstu@yandex.ru

Presented pilot studies of a hydrodynamic picture of movement of streams of liquid in mixing devices of a cylindrical and profiled form. The Shown efficiency of use of the device of a profiled form with the Helicoid type rotor when hashing fibrous suspensions of various concentration.

Keywords: hydrodynamics, homogeneity, case, the directing device, cavity, profiling, rotor, suspension, circulation.

\section{References}

1. Chichaev V.A., Glezin M.L., Ekimova V.A. Oborudovanie tselliulozno-bumazhnogo proizvodstva. V 2-kh tomakh. T. 2. Bumagodelatel'nye mashiny. [Equipment for pulp and paper production. In 2 v. Vol. 2. Paper machines.]. Moscow, 1981, 264 p. (in Russ.).

2. Fliate D.M. Tekhnologiia bumagi. [Paper technology.]. Moscow, 1988, 440 p. (in Russ.).

3. Ivanov D.A., Ivanov K.A., Rudenko A.P. Khimiia rastitel'nogo syr'ia, 2011, no. 4, pp. 319-324. (in Russ.).

4. Ivanov D.A., Ivanov K.A., Rudenko A.P. Lesnoi i khimicheskii kompleksy - problemy i resheniia: sb. statei vseros. nauch.-praktich. konf. [Forest and chemical complexes - problems and solutions: a collection of articles all-Russian scientific-practical conference.]. Krasnoyarsk, 2012, vol. II, pp. 46-50. (in Russ.).

5. Strenk F. Peremeshivanie i apparaty s meshalkami. [Stirring machines with agitators.]. Leningrad, 1975, 384 p. (in Russ.).

6. Patent 2453589. (RU). (in Russ.).

7. Terent'ev O.A. Gidrodinamika voloknistykh suspenzii v tselliulozno-bumazhnom proizvodstve. [Hydrodynamics fibrous suspensions in the pulp and paper industry.]. Moscow, 1980, 248 p. (in Russ.).

Received March 17, 2013

\footnotetext{
* Corresponding author.
} 